\title{
Diferencias de las declaraciones de los menores en función del sexo
}

\author{
Differences in the children's statements depending on the gender variable
}

\author{
Dolores Padilla-Racero \\ Universidad de Málaga, España
}

\begin{abstract}
Resumen
En los procedimientos de separación, surge el problema de determinar el tiempo de comunicación del progenitor que no ostenta la guarda y custodia de los hijos, con éstos. Esta determinación a veces va a estar supeditada al testimonio de los hijos menores El alcance del testimonio de los hijos va a depender de la credibilidad que el órgano judicial otorgue a los menores. Habiéndose demostrado que los menores tienden a decir la verdad, este estudio determina que el sexo no influye en la veracidad del testimonio de los menores ni en que sus testimonios se vean alterados por posibles manipulaciones.
\end{abstract}

Palabras clave: manipulación, síndrome de alienación parental, testimonio, sexo, menores.

\begin{abstract}
In the procedures of separation, there arises the problem of determining the time of communication of the progenitor who does not show the guard and custody of the children, with these. This determination sometimes is going to be subordinated to the testimony of the minor children. The scope of the testimony of the children is going to depend on the credibility that the judicial organ grants to the minors. Having been demonstrated that the minors stretch to tell the truth, this study determines that the sex does not influence the veracity of the testimony of the minors not in that his testimonies meet altered by possible manipulations.
\end{abstract}

Keywords: manipulation, parental alienation syndrome, testimony, sex, minor.

\section{Justificación teórica}

Si hablamos de la credibilidad del testimonio de los menores, varones y mujeres, dentro de los procedimientos judiciales de separación o divorcio, donde se dilucida con que progenitor van a convivir éstos y el régimen de comunicación con el progenitor con el que no van a convivir, tenemos que hablar del SAP, constructo formulado por el psiquiatra estadounidense Richard Gardner (1985). Vaya por delante, que a pesar de carecer de toda cientificidad el concepto, su aplicación como tal, es un hecho en el ámbito judicial. Gardner (1991, p.15) define este síndrome como: "Trastorno infantil que surge casi exclusivamente en el contexto de las disputas por la custodia de los niños. Su manifestación primaria es la campaña de denigración del niño contra un padre, una campaña que no tiene justificación”. Esta campaña de denigración de la que habla el SAP, conlleva la atribución de falsedad al testimonio de los menores, en el contexto de la disputa por la guarda y custodia o el régimen de visitas con éstos. Ello implica que, si el menor manifiesta que está siendo maltratado o abusado por uno de los progenitores, el SAP determina que el testimonio es falso y concomitantemente se dejará de investigar el posible abuso o maltrato. Autores como Vilalta (2011) reafirman la existencia del SAP, pero como explican Clemente y Padilla (2015) "Vilalta, al igual que Gardner, ahonda en la perspectiva de basarse en intuiciones y dejar de lado los datos".

El SAP que Gardner formuló se inspira en psicoanálisis de Freud: tanto uno como el otro son hipótesis no falsables, ya que tanto la aceptación como la no aceptación del diagnóstico suponen la afirmación de éste, el SAP interpreta que el menor miente cuando vilipendia al padre (normalmente el progenitor no custodio es el varón) y en la teoría psicoanalítica el menor fantasea cuando relata recuerdos de abuso sexual durante la infancia, según Gardner los niños pueden tener fuertes impulsos sexuales e iniciar ellos encuentros sexuales con los adultos, mintiendo y culpando luego al adulto si la relación es descubierta (Gardner, 1986, 1992) y Freud a través de la teoría edípica, nos traslada que el niño es un seductor capaz de matar a su padre para usurpar su lugar en relación a su madre. En suma, tanto el SAP como el psicoanálisis, se adscriben al campo de la ideología huyendo del de la ciencia. Clemente y Padilla (2015) demuestran con un estudio que los menores tienden a decir la verdad, por lo que partiendo de ello, en el presente estudio se abordará si el sexo es una variable mediadora en la veracidad del testimonio de los menores. Se va a hipotetizar que el sexo no influye en la veracidad del testimonio de los menores.

\section{Método}

\section{Participantes}

Se realiza un estudio en un colegio con 300 menores de ambos sexos y de edades comprendidas entre 6 y 12 años. 


\section{Instrumentos}

Mediante entrevista personal con cada niño se les pregunta sobre lo que han presenciado, consignándose los resultados en una hoja de respuesta por parte del equipo de investigación. La entrevista con cada niño es individual y al término de la misma, el niño no vuelve al aula donde esperan los que aún no han sido entrevistados para evitar la contaminación. Las variables dependientes van a ser los testimonios de los niños en las dos fases del experimento y las variables independientes serán el hecho de presenciar o no una agresión, la presión e imagen que se les proyecta a los niños sobre la parte actora de la agresión y el sexo de los niños.

\section{Procedimiento.}

Tras los pertinentes permisos a la dirección de un centro de educación primaria, se les explica a profesores y tutores el experimento, quienes explican $\mathrm{y}$ piden permiso $\mathrm{y}$ discreción a los padres. El experimento va a constar de dos fases que se van a desarrollar en tres días diferentes, estando los niños divididos en dos grupos:

- El primer día, un grupo va a presenciar una agresión verbal simulada y el otro no.

- El segundo día, los dos grupos van a ser preguntados, en el primer caso, para que relaten que ha ocurrido y en el segundo, se les va a preguntar si han visto algo problemático. Se utilizó un diseño con medidas post.

- El tercer día, se les pregunta a los dos grupos de niños sobre lo que han visto, pero informándoles previamente, de que la persona actora de agresión va a ser o no su tutora en el futuro e infiriéndoles una imagen positiva o negativa de la misma, creándose por tanto cuatro posibles grupos de información. El diseño es factorial $\mathrm{A}^{*} \mathrm{~B}$, $2 * 2$, solo con medidas post.

La información de lo que los niños han presenciado o no, se recoge dos veces, tras lo cual, se les comunica a los niños que la agresión no ha sido real, y que su fin era ver como ellos reaccionarían.

\section{Resultados}

El sexo de los menores no es una variable mediadora de la veracidad del testimonio de éstos. Los menores, tanto los varones como las mujeres, vierten testimonios veraces en el mismo porcentaje, tanto si presencian la agresión como si no. Sin embargo, el hecho de presenciar o no la agresión, sí es significativo en el índice de veracidad del testimonio de los menores: cuando éstos presencian la agresión, prácticamente todos dicen la verdad y cuando no la presencian, el índice de veracidad desciende notablemente.
Tabla 1: Información en función de la producción de una agresión verbal

\begin{tabular}{|c|c|c|c|c|c|c|}
\hline & & & \multicolumn{2}{|c|}{ Varones } & \multicolumn{2}{|c|}{ Mujeres } \\
\hline & & & Verdad & Mentira & Verdad & Mentira \\
\hline \multirow{4}{*}{$\begin{array}{l}\text { Tipo de } \\
\text { agresión }\end{array}$} & Agresión & Frecuencia $(\mathrm{N})$ & 66 & 5 & 66 & 5 \\
\hline & & Porcentaje & $93,0 \%$ & $7,0 \%$ & $93,0 \%$ & $7,0 \%$ \\
\hline & No & Frecuencia $(\mathrm{N})$ & 37 & 26 & 37 & 26 \\
\hline & agresión & Porcentaje & $58,7 \%$ & $41,3 \%$ & $58,7 \%$ & $41,3 \%$ \\
\hline \multirow[t]{2}{*}{ Total } & & Frecuencia $(\mathrm{N})$ & 126 & 103 & 103 & 31 \\
\hline & & Porcentaje & $77,8 \%$ & $76,9 \%$ & $76,9 \%$ & $23,1 \%$ \\
\hline
\end{tabular}

Otro dato reseñable es que, el testimonio de los menores varones y mujeres, no varía significativamente cuando se les intenta manipular introduciendo variables de presión e imagen, lo que implica que no son susceptibles de ser manipulados por una tercera persona. Cuando presencian la agresión, y luego se les intenta manipular, introduciendo variables de presión e imagen, prácticamente la totalidad de los menores, varones y mujeres, mantienen su testimonio, de hecho solo siete menores, de ellos, cuatro mujeres y tres varones, mienten respecto lo que han visto, frente a los 146 menores que se reafirman en su testimonio verídico.

Tabla 2: Efectos del grado de presión y de la imagen para los sujetos que presenciaron la agresión

\begin{tabular}{|c|c|c|c|c|c|c|c|}
\hline \multirow[t]{2}{*}{ Fase 2} & & & & \multicolumn{2}{|c|}{$\begin{array}{l}\text { Presión } \\
\text { varones }\end{array}$} & \multicolumn{2}{|c|}{$\begin{array}{l}\text { Presión } \\
\text { mujeres }\end{array}$} \\
\hline & & & & Alta & Baja & Alta & Baja \\
\hline \multirow[t]{6}{*}{ Verdad } & Información & $\begin{array}{l}\text { Buena } \\
\text { persona }\end{array}$ & $\begin{array}{l}\text { Frecuencia } \\
(\mathrm{N}) \\
\end{array}$ & 21 & 14 & 18 & 17 \\
\hline & & & Porcentaje & 60,0 & 40,0 & 51,4 & 48,6 \\
\hline & & & & $\%$ & $\%$ & $\%$ & $\%$ \\
\hline & & $\begin{array}{l}\text { Mala } \\
\text { persona }\end{array}$ & $\begin{array}{l}\text { Frecuencia } \\
(\mathrm{N}) \\
\end{array}$ & 24 & 21 & 19 & 12 \\
\hline & & & Porcentaje & 53,3 & 46,7 & 61,3 & 38,7 \\
\hline & & & & $\%$ & $\%$ & $\%$ & $\%$ \\
\hline \multirow[t]{3}{*}{ Mentira } & Información & $\begin{array}{l}\text { Mala } \\
\text { persona }\end{array}$ & $\begin{array}{l}\text { Frecuencia } \\
(\mathrm{N}) \\
\end{array}$ & & 3 & 3 & 1 \\
\hline & & & Porcentaje & & 100,0 & 75,0 & 25,0 \\
\hline & & & & & $\%$ & $\%$ & $\%$ \\
\hline
\end{tabular}


Tabla 3: Efectos del grado de presión y de la imagen para los sujetos que no presenciaron la agresión

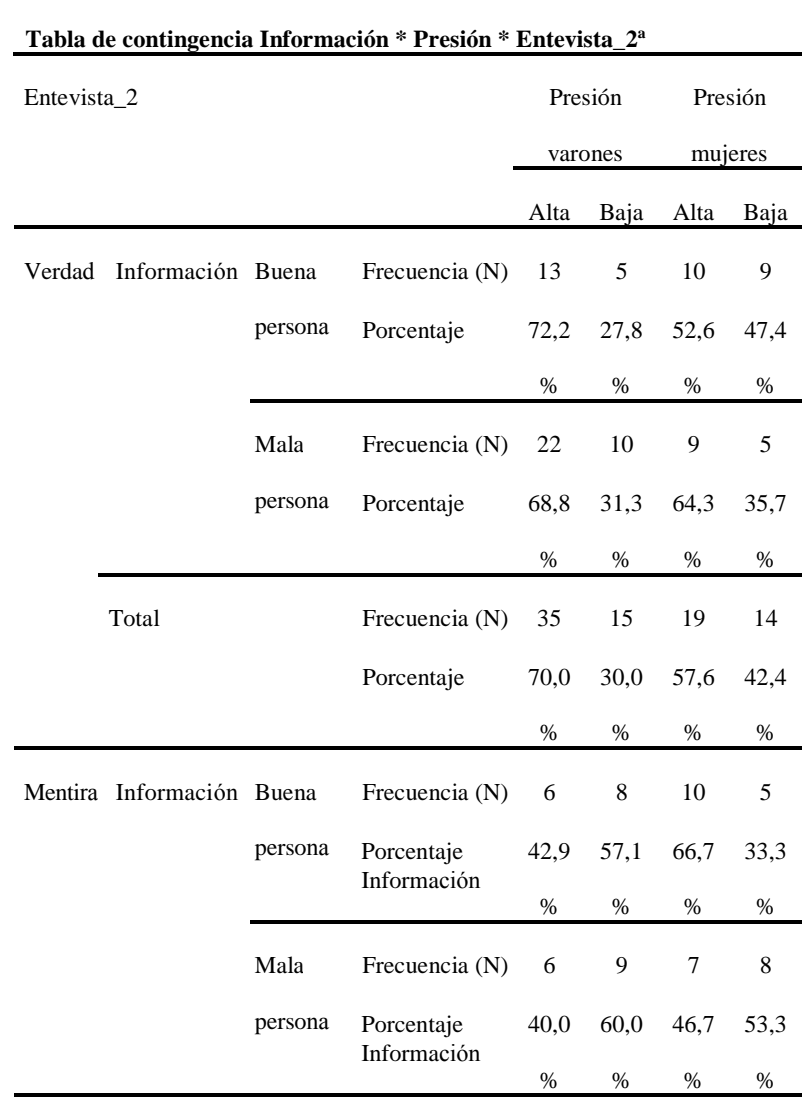

\section{Discusión}

Tanto los menores varones como las menores mujeres tienden a decir la verdad. En el caso de que presencien algo conflictivo, dicen la verdad en un porcentaje amplísimo cercano a la totalidad. Cuando no presencian ningún episodio conflictivo, pero se les infiere una pregunta sugestiva a cerca de lo que han presenciado, tanto varones como mujeres, en un $40 \%$ fabrican un recuerdo. Este recuerdo es compatible con un fenómeno presente en el funcionamiento de la memoria de todo ser humano y que no constituye ninguna patología: la falsa memoria. El concepto de la falsa memoria se introdujo en la psicología tras las investigaciones de Loftus, Miller y Burns (1978) La veracidad del testimonio de los menores varones y mujeres, además no se altera cuando se les intenta manipular diciéndoles que en el futuro van a depender de la persona sobre las que tienen que informar ni cuando se les proyecta una determinada imagen sobre esta persona.

Por tanto, se demuestra que las ideas de Gardner no tienen soporte empírico, el presente estudio demuestra lo contrario. Uno de los axiomas del SAP es que el niño es mentiroso por naturaleza y que es capaz de sostener una mentira con tal de perjudicar a un tercero (el progenitor no custodio), sin embargo los datos demuestran que los menores, tanto varones como mujeres, dicen la verdad cuando presencian un hecho y pueden fabricar un recuerdo cuando no lo presencian, como el resto de seres humanos. Y este recuerdo fabricado, gira en torno a la interacción de los menores entre ellos mismos o entre ellos y la tarea o actividad, no en el lanzamiento de acusaciones de hechos conflictivos atribuidos a terceras personas. El menor pues, no es mentiroso por naturaleza. Al autodefinirse como síndrome, el SAP lo hace alegando que en la campaña de denigración que el menor hace contra el progenitor no custodio, están presentes además de la manipulación del progenitor con el que el menor convive, las propias fabricaciones del menor. Asentado ya que el menor no miente, los resultados del estudio, demuestran además que la premisa de la facilidad de la manipulación sobre los menores también es falsa. Tanto el testimonio de los menores varones como mujeres no se ve alterado cuando a éstos se les plantea la posibilidad de dependencia, en una relación de asimetría, de la persona actora de la agresión y además de les proyecta una determinada imagen de ésta. La introducción de estas variables de presión e imagen en el diseño no es baladí: recrean por un lado, la situación de asimetría que se da entre el menor y el progenitor con el que convive y al que Gardner atribuye la manipulación sobre el menor y la imagen negativa que supuestamente este progenitor infiere al menor sobre el progenitor no custodio. Por tanto, esa falsa memoria, presente en los menores varones y mujeres que no ven la agresión, no está sujeta a variables de presión e imagen, como preconiza el SAP.

Los resultados del presente estudio refutan una de las ideas principales del SAP que Gardner propuso, la de que los niños mienten y son fácilmente manipulables, capaces además de mantener una mentira con el fin de perjudicar a un tercero y verifican en contraposición, las teorías de autores como Clemente (2013), quien afirma que "el SAP es un atentado contra la ciencia, contra el Estado de Derecho, y contra los menores y sus progenitores. De hecho, una teoría no científica no debería ser utilizada dentro del sistema de administración de Justicia, ya que en ese caso el primer perjudicado será el menor" y Escudero et al. (2010, p. 22) quienes sostienen que "no existe dicha acreditación sobre la metodología, y el constructor del supuesto PAS no puede garantizar en modo alguno la falsedad o no de las denuncias; si pueden existir múltiples explicaciones válidas sobre el rechazo del menor hacia el progenitor las cuales el supuesto PAS no pueda descartar; si la posibilidad de falsos positivos (determinar erróneamente que un menor tiene un supuesto PAS sin tenerlo) es tan elevado que en tal caso la medidas de cambio de custodia pueden suponer realmente un riesgo y daño inasumible para el menor; y si pudiéndose demostrar que los fundamentos del supuesto PAS se han construido falseando la terminología empleada y establecido una lógica que permita justificar cualquier resultado a priori, ¿cabría entonces que un sistema de protección como es el judicial permitiese mantener su uso?”. Este estudio pone de manifiesto la falsedad de la terminología empleada para construir el SAP, ya que 
refuta uno de los pilares del mismo: el de que el niño es un mentiroso por naturaleza y fácilmente manipulable. Escudero et al. (2008) considera por todo ello que el sistema judicial debe revisar el empleo del PAS y sus medidas, que bajo el reclamo de «terapéuticas» solo pueden generar daño psíquico y perversión del uso de la ciencia.

Como conclusión final, decir que el sistema legal ha de tomar en su debida consideración el testimonio de los menores, tanto varones como mujeres, pues se demuestra que son testimonios veraces, sujetos a las mismas dinámicas de los testimonios de los adultos en cuanto a lo que a la falsa memoria se refiere, sin poder atribuir al testimonio de los menores varones y mujeres la falsedad y animosidad que el SAP categóricamente les atribuye.

Como limitaciones más destacadas al presente estudio destacar, que se ha llevado a cabo con una muestra de niños que no están sometidos a la situación conflictiva en la que se desarrolla el concepto del SAP que se ha estudiado, y ello por razones de tamaño de la muestra necesaria para que arrojase resultados estadísticos significativos. Otra limitación sería que mientras que el estudio se ha hecho en un rango de edades desde los 6 a los 12 años y en la realidad, bajo los criterios del SAP, la veracidad del testimonio de los menores está en entredicho desde que se disuelve la unidad familiar hasta que el menor alcanza la mayoría de edad. Por último, la muestra sobre la que se hace el presente estudio ha podido presenciar una agresión verbal, que por motivos éticos no llega a ser de agresión física o sexual, como pueden ser presenciadas por los menores en la realidad.

\section{Referencias bibliográficas}

Clemente, M. (2013). El Síndrome de alienación parental: un atentado contra la ciencia, contra el estado de derecho, y contra los menores y sus progenitores. Infancia, Juventud y Ley, 4, 48-57.

Clemente, M. (2014). Aproximaciones psicológicas y jurídicas a la guarda y custodia. Madrid: Síntesis.

Clemente, M. \& Padilla-Racero, D. (2015). Are children susceptible to manipulation? The best interest of children and their testimony. Children and Youth Services Review, 51, 101-107.

DOI:

10.1016/j.childyouth.2015.02.003.

Escudero et al. (2008). La Lógica del Síndrome de Alienación Parental de Gardner (SAP): Del Síndrome "Puro" a la "Terapia de la Amenaza". Revista de la Asociación Española de Neuropsiquiatría, 28 (2), 285-307.
Escudero. A.; González, D.; Méndez, R.; Naredo, C.; Pleguezuelos, E.; Vaccaro, S. \& Pérez, A.M. (2010). Informe del grupo de trabajo de investigación sobre el llamado síndrome de alienación parental. Madrid: Ministerio de Sanidad, Política Social e Igualdad.

Gardner R. (1985). Recent trends in divorce and custody litigation. Academy Forum, 29, 3-7.

Gardner, R.A. (1986). Child Custody Litigation: A Guide for Parentsand Mental Health Professionals. Cresskill, NJ: Creative Therapeutics.

Gardner, R. A. (1991). Legal and Psychotherapeutic Approaches to the Three Types of Parental Alienation Syndrome Families. When Psychiatry and the Law Join Forces. Court Review, 28 (1), pp. 14-21.

Gardner, R.A. (1992). True and False Accusations of Child Sex Abuse. Cresskill, NJ: Creative Therapeutics

Loftus, E. F., Miller, D. G. \& Burns, H. J. (1978). Semantic integration of verbal information into a visual memory. Human Learning and Memory, 4, 19-31.

Vilalta, R.J. (2011). Descripción del Síndrome de Alienación Parental en una muestra forense. Psicothema, 23 (4), 636-641 